\title{
A New Era of Analysis with Spherical-Aberration-Corrected STEM - Atomic and Electronic Information Approaching the Single Atom Level
}

\author{
R. Erni, P. Tiemeijer, S. Kujawa, M. van der Stam, M. Stekelenburg, B. Freitag \\ FEI Company, Building AAE, Achtsteweg Noord 5, Eindhoven, The Netherlands
}

A corrector for the spherical aberration in the probe-forming optical part of a transmission electron microscope allows for increasing the spatial resolution in scanning transmission mode [1]. By collecting electrons scattered to high angles, atomic-number $(Z)$ sensitive maps can be recorded - at a resolution beyond the one-Ångström barrier. Such Z-contrast micrographs make it possible to extract direct structural information of materials and their defects on the atomic level.

Besides the high spatial resolution feasible with a probe $C_{\mathrm{S}}$ corrector, electron probes of high current can be formed. The increased probe current is particularly beneficial for analytical work on the atomic scale. Recent developments of electron optical devices have also lead to the realization of electron monochromators [2]. An electron monochromator implemented between the electron source and the illumination system reduces the energy spread of the incident electron beam. Apart from improving the information limit in HRTEM, an electron monochromator significantly enhances the information content in electron energy-loss spectra; electron energy-loss spectroscopy can ultimately be performed with an energy resolution approaching $100 \mathrm{meV}$ at $300 \mathrm{keV}$ primary energy.

The integration of an electron monochromator AND a probe $C_{\mathrm{S}}$ corrector in one system allows for combining their benefits. This combination has been realized on a FEI Titan system equipped with a Wien-filter type monochromator and a hexapole probe $C_{\mathrm{S}}$ corrector [3] operated at 300 and $200 \mathrm{kV}$. While the monochromator enables a high energy resolution in EELS, more than counterbalancing the loss of current induced by the monochromator, the $C_{\mathrm{S}}$ corrector warrants that a small electron probe of high current can be formed. High energy-resolution electron energy-loss spectroscopy on the atomic scale has thus become feasible.

This advanced analytical capability in STEM will certainly touch physical limits in materials science. For HR-EELS requires a high dispersion of the spectrometer, the electron dose needed to record a signal of sufficient signal-to-noise ratio has to be larger than in conventional EELS working at lower dispersions. The electron dose a single atomic column can stand is therefore a critical value. Another limiting factor to consider is the localization of the EELS signal. The localization of inelastic electron scattering is controversial; performing electron energy-loss spectroscopy with an atomic-size monochromated electron probe will help to reveal the ultimate spatial resolution which can be achieved in core-loss and valence-loss electron energy-loss spectroscopy.

In this contribution, utilization examples of probe $C_{\mathrm{S}}$ corrector and monochromator and their combination will be described. The combination of these technologies is anticipated to opening doors for researchers to study morphology, crystallography, elemental and chemical composition as well as electronic structure at resolution levels not demonstrated before in one instrument. 
The authors thank the involved employees of CEOS $\mathrm{GmbH}$ for their excellent contributions to making the next generation of corrector technology on Titan a success. The Titan platform is the basis of the TEAM project of the USA Department of Energy with the goal to arrive at 0.5 Ångström resolution in TEM and STEM on a single system, in combination with a Cc corrector developed in co-operation with CEOS GmbH.

\section{References}

[1] P.E. Batson et al., Nature 418 (2002) 617.

[2] P.C. Tiemeijer, Inst. Phys. Conf. Ser. 161 (1999) 191.

[3] M.Haider, et.al, Ultramicroscopy 81 (2000) 163

[4] M.J.Franssen et.al, Adv. Imag. Elec. Phys 111 (1999) 91

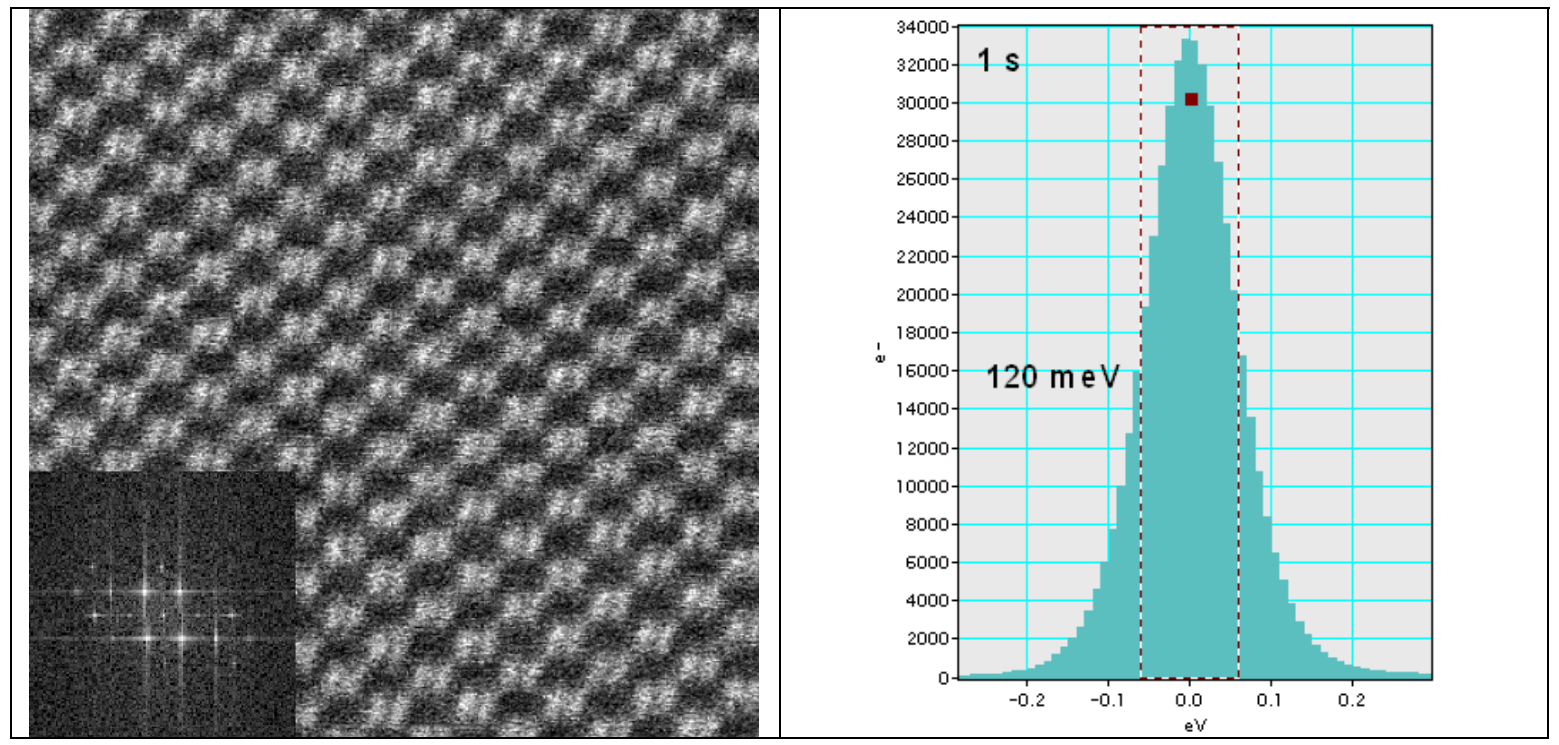

Fig. 1. Probe Cs corrected and monochromized Titan -uniquely designed for the Ernst-RuskaCentrum in Jülich, Germany- operated at $300 \mathrm{kV}$. The Si [110] shows STEM resolution down to $0.105 \mathrm{~nm}$ and the probe used has a FWHM of $\Delta \mathrm{E}=0.14 \mathrm{eV}$ at $1 \mathrm{~s}$ exposure time and a probe current of $60 \mathrm{pA}$ (19mrad acceptance angle).

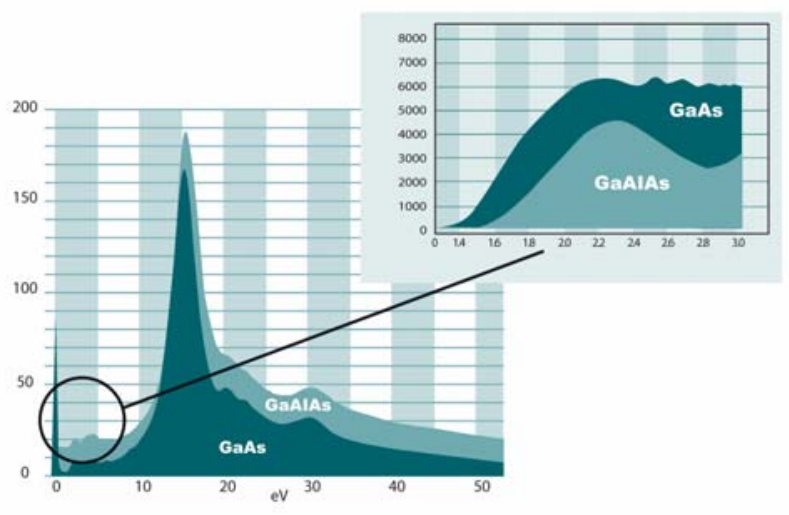

Fig. 2. An application example of Low-loss EELS using Titan's monochromator. The EELS spectrum of GaAs and GaAlAs shows the band gap next to the zero loss peak. The insert shows the shift of the band gap of GaAs and GaAlAs. 